\title{
Human aldo-keto reductases: structure, substrate specificity and roles in tumorigenesis
}

\author{
Jun Ma and Deliang Cao* \\ Department of Medical Microbiology, Immunology and \\ Cell Biology, Simmons Cancer Institute, Southern Illinois \\ University School of Medicine, 913 N. Rutledge Street, \\ Springfield, IL 62794, USA \\ * Corresponding author \\ e-mail: dcao@siumed.edu
}

\begin{abstract}
The aldo-keto reductase (AKR) superfamily consists of over 150 protein members sharing similar structure and enzymatic activities. To date, 13 human AKRs have been identified, and they participate in xenobiotic detoxification, biosynthesis and metabolism. Increasing evidence suggests the involvement of human AKR proteins in cancer development, progression and treatment. Some proteins demonstrate multiple functional features in addition to being a reductase for carbonyl groups. This review article discusses the most recent progress made in the study of humans AKRs.
\end{abstract}

Keywords: aldo-keto reductases; AKR1B1; AKR1B10; AKR1C; anticancer agents; carbonyls; polycyclic aromatic hydrocarbons; prostaglandins; retinaldehyde; tumorigenesis.

\section{Introduction}

Aldo-keto reductases (AKRs) are a large group of NAD $(\mathrm{P}) \mathrm{H}-$ dependent protein enzymes with structural similarities. They are evolutionarily related and pervasively exist in a wide range of organisms, from bacteria to humans $(1,2)$. Over 150 AKRs have been identified thus far and a web page (http:// www.med.upenn.edu/akr/) supported by Dr. Penning holds the most updated information on this protein superfamily.

According to their sequence homology, AKR proteins are divided into 15 families $(1,3,4)$. Proteins with more than $40 \%$ sequence identity are grouped into a family and those sharing over $60 \%$ identity are categorized into a subfamily. A nomenclature system of AKR proteins designates a number to identify a family, a letter to indicate a subfamily, and then another number following the letter to denote a unique protein (4). For instance, aldo-keto reductase family $1 \mathrm{mem}$ ber B1 (AKR1B1) indicates that this protein belongs to family 1 , subfamily $B$ of the AKR superfamily, and it is the protein no. 1 in the subfamily $B$. In addition to the designation in this nomenclature system, some proteins in the AKR superfamily have different terms given by researchers.
For example, AKR1B1 is also known as aldose reductase (AR), and AKR1B10 is also referred to as aldose reductaselike-1 (ARL-1). Table 1 summarizes the AKR proteins expressed in humans, which fall within three families (AKR1, AKR6 and AKR7).

AKRs are mainly cytosolic monomeric protein enzymes with molecular weights at $34-37 \mathrm{kDa}$. AKRs use pyridine nucleotides (NADH or NADPH) as cofactors and catalyze a metabolic oxidation reduction, reducing carbonyl (aldehydic and ketonic) groups into corresponding alcoholic forms. There are two exceptions for the AKR proteins. First, not all AKR proteins are an enzyme. For instance, Rho (AKR1C10) and RhoB crystallins (aldose reductase-related protein) are major components of frog and gecko lens. They retain amino acid residues required for catalytic activity and bind to pyridine nucleotides, but have not or very limited enzyme activity towards general AKR substrates $(5,6)$. Similarly, human AKR6 proteins are channel proteins, controlling $\mathrm{K}^{+}$ion conductance, but do not have enzymatic activity. Second, not all AKR proteins are monomeric. For example, AKR6 $\beta$-subunits form a tetramer at the base of the $\mathrm{K}^{+}$channel, and AKR7A1 and AKR7A4 in rat form either homo- or heterodimers (7). This review article focuses on the discussion of human AKRs, especially on their intriguing roles in cancer development, progression and therapeutics.

\section{Structure and enzymatic activity of AKRs}

A public protein data bank (PDB; http://www.pdb.org/pdb/ home/home.do) lists 84 crystal structures from over 25 AKR proteins, and several are demonstrated for the binary [E$\mathrm{NAD}(\mathrm{P}) \mathrm{H}]$ and/or ternary [E-NAD $(\mathrm{P}) \mathrm{H} /$ substrate] complexes, where $\mathrm{E}$ indicates enzyme (an AKR protein). A $(\beta / \alpha)_{8}$ barrel motif represents the common crystal feature of AKR protein (Figure 1) $(8,9)$. The hydrophile outer envelope of eight external $\alpha$-helices embraces the eight inner parallel hyperboloid $\beta$-strands, and these 'rigid' structure elements are linked together by 'soft' loops. This motif has extensive functional utility, such as binding phosphate groups or metals, forming active catalytic interfaces or acting as a gated barrel for channeling reaction intermediates. Our laboratory found that AKR1B10 associates with acetyl-CoA carboxylase- $\alpha$ (ACCA) and prevents its ubiquitin-dependent degradation in human breast and colon cancer cells and, interestingly, this association does not affect the enzymatic activity of AKR1B10 (10). This physiological phenomenon could benefit from this structural feature of AKR1B10. Factually, owing to the advantages, this featured motif is present 


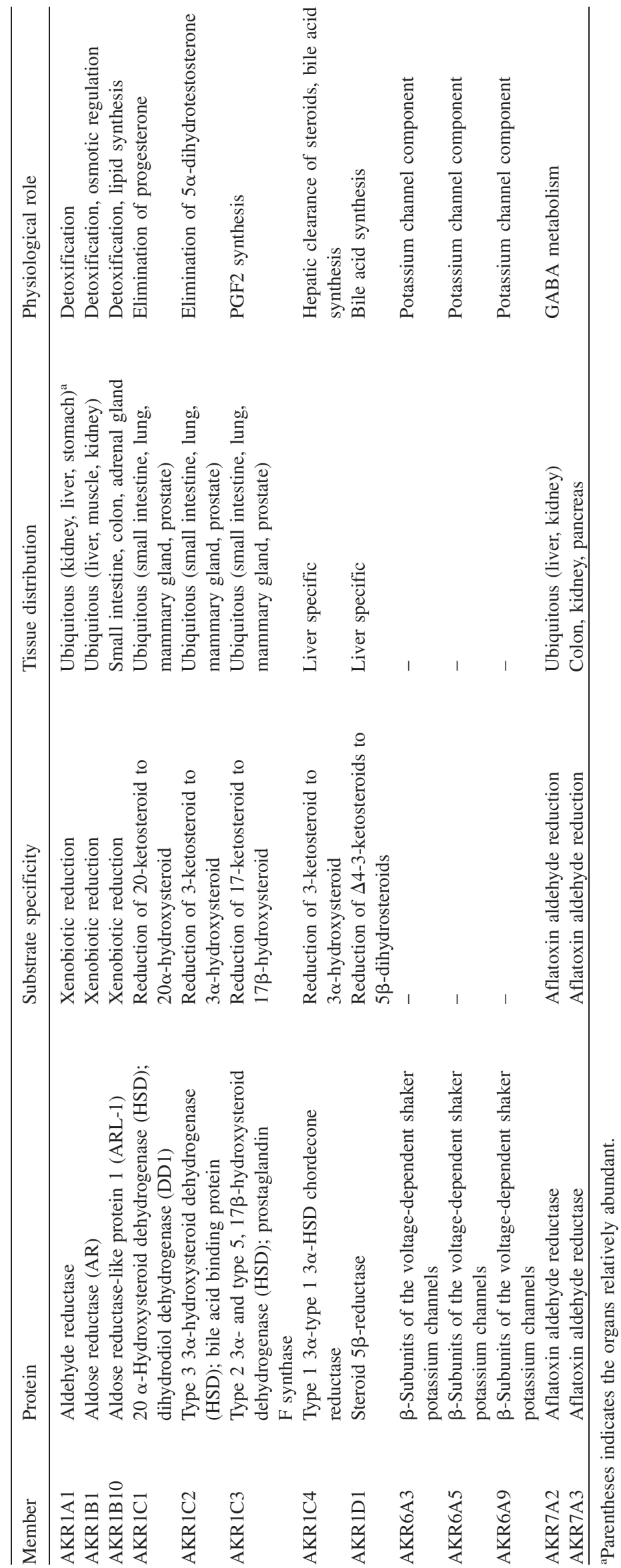




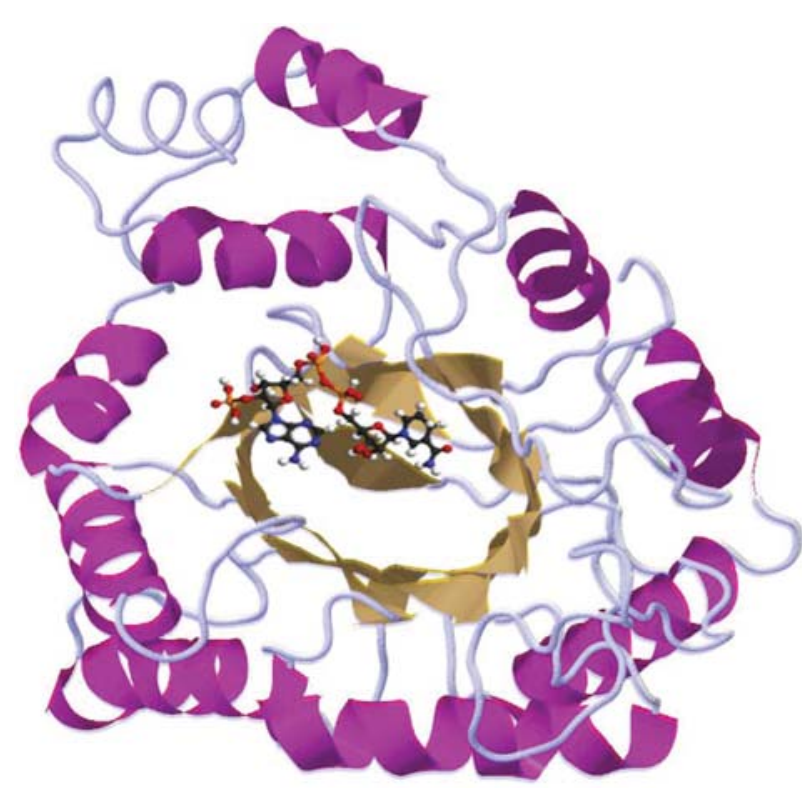

Figure 1 Crystal structure of the AKR1B1·NADPH binary complex.

The structure was downloaded from the RCSB Protein Database (ID\#: $1 \mathrm{ABN}$ ). The ribbon drawing is a bottom-view representation of the protein with NADPH bound to the active site.

in up to $10 \%$ of proteins in mammals (11). The active site in this motif of AKR proteins is located at the C-terminus, which is considered as the evidence of a common ancestry (12). In fact, this structure is particularly adaptive for the evolution of new function.

Although the $\alpha$-helix and $\beta$-strand structure is conservative among AKR proteins, diversity in amino acid sequence and/or peptide length exists in connective loops. This diversity results in variations in the arrangement of $\alpha$-helices, but the inner $\beta$-strand barrel is relatively constrained. As a result, binding and catalysis can be varied without effects of the basic protein structure. The loop located between the seventh and eighth $\beta$-strands of the barrel is a 'hot spot' of such variations. This region is diversified in the loop length and the number of additional helices, offering identity to individual families. The variations of loop length also occur among the members in the same subfamily. For example, aldehyde (AKR1A1) and aldose (AKR1B1) reductases have a long loop between $\beta 9$ and $\alpha 7$, which opens and closes upon binding or releasing of the cofactor, thus facilitating efficient binding to NADPH (13). However, in some AKRs, such as hydroxysteroid dehydrogenases (AKR1Cs), the shorter loop results in the absence of opening or closing movements (14).

In an oxidation reduction reaction, the pyridine nucleotide cofactor and carbonyl substrate bind to two different regions of the protein and converge at the active site. Most AKRs prefer binding NADPH over NADH as a reducing cofactor (15), but exceptions exist. For instance, AKR1C12 and AKR1B7 (also named MVDP) favor NADH rather than NADPH (16). The structural basis of NADPH preference is related to the positively charged lysine and arginine residues that bind to pyrophosphate backbone and 5'-phosphate group of pyridine nucleotide. The pyridine nucleotide cofactor binding site is relatively conserved, whereas the substrate binding cavity shows variations, which is largely defined by the residues from loops. Nevertheless, the active site is highly conserved across the whole family proteins. The conserved catalytic tetrad consists, such as AKR1B1, of Tyr-48, His110, Lys-77 and Asp-43 (17-22). They form an oxyanion binding site with the nicotinamide ring of the cofactor via a hydrogen bonding network.

Most AKR proteins are enzymes catalyzing the NAD $(\mathrm{P}) \mathrm{H}-$ dependent reduction of aldehydes and ketones to their corresponding alcoholic forms. Reduction catalyzed by AKRs proceeds in two steps: hydride ion transfer from NAD $(\mathrm{P}) \mathrm{H}$ to the carbonyl substrate and proton addition from the solvent for reduction of the carbonyl to alcohol (Figure 2). Hydride transfer is 4-pro- $R$ specific and the acceptor carbonyl group is polarized by the conserved active site in the tetrad, which acts as a general acid base for the reaction (23, 24). Mutation analysis suggested that the acid base catalytic group in AKRs is Tyr-48 in most cases. This tyrosine is universally conserved in all AKRs, whereas His-110 is not (25, $26)$. During the reaction, $\mathrm{NAD}(\mathrm{P}) \mathrm{H}$ cofactor binds first and leaves last. Transient kinetic studies showed that significant conformational changes occur upon binding of the cofactor to form a tight binary complex. Conformational changes during the release of cofactor partially or completely determine the overall turnover rate $(23,24)$, but in AKR1C ketosteroid reductases, the rate of chemical conversion and steroid product release also contribute to the turnover rate $(27,28)$. AKRs have broad diversity of substrates, which includes most biologic aldehydes and ketones generated endogenously during metabolism or from environment, such as food components, drugs or toxins. Therefore, AKRs are considered as protective enzymes responsible for xenobiotic detoxification.

AKR activity can be inhibited by small chemicals through interaction with the active site. For example, most tight-binding aldose reductase inhibitors (ARIs) have a polar group, usually a carboxylate, tethered from a hydrophobic core constructed by one or more ring structures. Inhibitors bind with their polar head group oriented close to the pyridine ring, forming hydrogen bonds with residues Tyr-48, His-110 and Tyr-111. Extensive hydrophobic interactions between inhibitors and AKR residues that line the deep hydrophobic active cavity help to stabilize the ternary enzyme-coenzyme-inhibitor complex $(29,30)$. In the past years, ARIs have been developed as therapeutic agents of diabetic complications $(31,32)$.

\section{$E \Leftrightarrow E^{*} \cdot N A D P H \Leftrightarrow E^{*} \cdot N A D P H \cdot S \Leftrightarrow E^{*} \cdot N A D P^{+} \cdot P \Leftrightarrow E \cdot N A D P^{+} \Leftrightarrow E$}

Figure 2 Kinetic mechanism of carbonyl reduction catalyzed by AKR proteins.

Binding of NADPH causes a conformational change of aldo-keto reductase (AKR) proteins (E to $\mathrm{E}^{*}$ ) in order to bind substrate (S). The alcoholic product is formed by transfer of the hydride ion from NADPH and addition of proton from the solvent to the carbonyl substrate. After the alcoholic product $(\mathrm{P})$ is released, the second conformation change occurs (E* to E) to release $\mathrm{NADP}^{+}$. 


\section{Human AKRs}

In humans, 13 different AKR proteins have been identified that fall into three different AKR families: AKR1, AKR6 and AKR7. The AKR1 family includes AKR1A1 (aldehyde reductase), AKR1B1 (aldose reductase), AKR1B10 (aldose reductase-like-1, also named small intestine aldose reductase), AKR1C1 [20 $\alpha$-hydroxysteroid dehydrogenase (HSD)/ dihydrodiol dehydrogenase (DD1)], AKR1C2 (type $33 \alpha-$ HSD/bile acid binding protein), AKR1C3 (type $23 \alpha-\mathrm{HSD}$ and type 5 17 $\beta$-HSD/prostaglandin F synthase), AKR1C4 (type $13 \alpha-\mathrm{HSD} /$ chordecone reductase) and AKR1D1 (steroid $5 \beta$-reductase); the AKR6 family contains $\beta$-subunits of the voltage-dependent shaker potassium channels (AKR6A3, AKR6A5 and AKR6A9); and the AKR7 family comprises aflatoxin aldehyde reductases AKR7A2 and AKR7A3 (Table 1).

In the human genome, AKR members belonging to the same subfamily (except for Kv $\beta$ in AKR6) usually form a gene cluster located on the same chromosome and share similar gene structures. The AKR1A and AKR1D subfamilies have only one member (AKR1A1 or AKR1D1) in humans, and the gene is located on chromosome 1p32-33 and 7q3233, respectively (Table 2). The AKR1B1 and AKR1B10 gene are located closely on chromosome 7q33-35 and contains 10 exons each. The four human AKR1C genes, consisting of nine exons, form a distinct cluster on chromosome 10p14-15. The two human aflatoxin reductase genes, AKR7A2 and AKR7A3, reside on chromosome 1p35.1-36.23 and are composed of seven exons. Genes of the AKR6 family (three $\mathrm{Kv} \beta$ genes) are different from other AKRs in several aspects. For example, these genes do not form a cluster on a chromosome, but are located on chromosomes 1, 3 and 17, respectively. In addition, despite the similarities in intron/ exon structures, the gene length varies from $7.0 \mathrm{~kb}$ for AKR6A9, Kvß3 to $416 \mathrm{~kb}$ for AKR6A3, Kv $\beta 1$.

\section{Tissue distribution of human AKRs}

AKR1A1 is a general metabolic enzyme that is involved in the reduction of glyceraldehyde to glycerol and melvadate to mevalonic acid, playing a central role in triglyceride and cholesterol biosynthesis $(33,34)$. This enzyme is ubiquitously expressed in most tissues with the highest level in the kidney proximal tubules (35). AKR1A1 is also highly expressed in brain, which is consistent with its role in the metabolism of aldehydes derived from monoamine oxidase (36). AKR1B1 that catalyzes the first step in the polyol pathway is also broadly expressed with high expression levels in the liver, skeletal muscle, cardiac muscle, kidney, ovary, testis, prostate and small intestine $(37,38)$. By contrast, AKR1B10 shows restricted tissue distribution, predominately expressed in the small intestine, colon and adrenal gland $(39,40)$. This specific distribution of AKR1B10 could be functionally related to its efficient detoxification of dietary or lumen-microbial $\alpha, \beta$-unsaturated carbonyls (41-43). Four AKR1C proteins in humans that share over $86 \%$ amino acid sequence homology are found in the liver, but have different extrahepatic distributions. AKR1C4 is expressed predominantly in the liver, but AKR1C1-AKR1C3 are highly expressed in the small intestine, lung, mammary gland and prostate, which reflect their roles in xenobiotic and steroid metabolism $(44,45)$. AKR1D1 (also known as steroid $5 \beta$-reductase) appears to be liver specific owing to its role in bile acid biosynthesis and steroid hormone clearance $(46,47)$. Similar to AKR1A1 and AKR1B1, AKR7A2 is widely expressed in human tissues with a high level in the liver, small intestine, kidney and cerebrum. Its distribution in brain is consistent with its role in the metabolism of $\gamma$-aminobutyric acid (GABA) metabolite succinic semialdehyde (48). AKR7A3 was cloned and identified from the liver, but is primarily expressed in the stomach, colon, kidney and pancreas (49).

\section{Substrate specificity and pathophysiological roles of human AKRs}

Aldehydes and ketones are produced endogenously, e.g., 4hydroxynonenal (HNE) and acrolein derived from lipid peroxidation, glyoxal and methylglyoxal from sugar metabolism, and succinic semialdehyde from GABA. Exogenous carbonyl compounds are present in diet (e.g., 2-hexenal in

Table 2 Chromosome locations and structures of human AKR genes.

\begin{tabular}{|c|c|c|c|}
\hline Gene & Chromosomal localization & Exon number & Access number \\
\hline akrlal & $1 \mathrm{p} 33-\mathrm{p} 32$ & 8 & J04794 \\
\hline$a k r 1 b 1$ & $7 q 35$ & 10 & J04795 \\
\hline$a k r 1 b 10$ & $7 q 33$ & 10 & AF052577 \\
\hline$a k r l c 1$ & $10 \mathrm{p} 15-\mathrm{p} 14$ & 9 & D26124 \\
\hline$a k r l c 2$ & $10 \mathrm{p} 15-\mathrm{p} 14$ & 9 & L32592 \\
\hline akrlc3 & $10 \mathrm{p} 15-\mathrm{p} 14$ & 9 & $L 43839$ \\
\hline akrlc4 & $10 \mathrm{p} 15-\mathrm{p} 14$ & 9 & M33375 \\
\hline akrldl & $7 q 32-q 33$ & 7 & Z28339 \\
\hline$a k r 6 c 3$ & $3 q 26.1$ & 7 & U33428 \\
\hline akr6c5 & $1 \mathrm{p} 36.3$ & 13 & U33429 \\
\hline akr6c9 & $17 \mathrm{p} 13.1$ & 13 & AF016411 \\
\hline$a k r 7 a 2$ & $1 \mathrm{p} 35.1-\mathrm{p} 36.23$ & 9 & AF026947 \\
\hline$a k r 7 a 3$ & $1 \mathrm{p} 35.1-\mathrm{p} 36.23$ & 13 & AF040639 \\
\hline
\end{tabular}


vegetables and diacetyl in butter and wine) and environment (e.g., chloroacetaldehyde from vinyl chloride and transtrans-muconaldehyde from benzene). These unsaturated chemicals are highly electrophilic and can interact with proteins, nucleic acids, and other macromolecules, inducing cell damage and apoptosis $(41,42,50-52)$. Therefore, unsaturated carbonyls are highly mutagenic and tumorigenic. AKRs in humans, therefore, can play a primary detoxicant role by reducing them to less toxic alcoholic forms. AKR1A1 and AKR1B1 are ubiquitously expressed in human tissues and show a broad range of substrate specialty. They show high $k_{\text {cat }} / K_{\mathrm{m}}$ values in reducing aliphatic aldehydes (e.g., succinic semialdehyde, 1,2-naphthoquinone and 16-ketoestrone), aromatic aldehydes (e.g., carboxybenzaldehyde and nitrobenzaldehyde), aromatic ketones (e.g., nitroacetophenone), $\alpha, \beta$ unsaturated ketones (e.g., acetone and HNE) and dicarboyl (e.g., methylglyoxal and hexanedione) $(33,53)$. These two enzymes most probably act as universal protective enzymes against carbonyls. In addition, AKR1B1 is considered as a causative factor for diabetic complications by converting glucose to sorbitol under hyperglycemia. This is a rate-limiting step of the polyol pathway, and the polar sorbitol accumulates inside cells and induces osmolytic pressure and oxidative stress. Sorbitol accumulation has been observed in cataractous lens, Schwann cells and other diabetic lesion tissues; and transgenic mice with AKR1B1 overexpression show high susceptibility to diabetic cataractogenesis and neuropathy $(37,53)$.

AKR1B10 with primary expression in intestine and colon displays strong catalytic activity to $\alpha, \beta$-unsaturated carbonyls which originated from lipid peroxidation and/or diets, such as acrolein, crotonaldehyde, HNE, trans-2-hexanal and trans-2,4-hexadienal (39). Owing to the feature of constant exposures of the intestine to intracellular, dietary and lumenmicrobial carbonyls, the intestine-specific expression of AKR1B10 could render it a role as a chemical-preventive barrier in the intestinal tract. In addition, recent studies from our laboratory have shown that AKR1B10 associates with ACCA, a rate-limiting enzyme in fatty acid biosynthesis, and mediates its ubiquitin-dependent degradation, thus regulating fatty acid synthesis and cell growth and survival $(10,54)$.

AKR1C1-C4 proteins are poor catalysts of aromatic aldehydes, aldoses or dicarbonyls, but more affinitive to steroid hormones, prostaglandins, polycyclic aromatic hydrocarbons (PAHs) and trans-dihydrodiols $(44,45)$. Recombinant AKR17A2 shows narrow specificity towards succinic semialdehyde, 2-nitrobenzaldehyde, pyridine-2-aldehyde, 1,2-naphthoquinone and isatin, suggesting its role in the detoxification of these metabolites. Among these, succinic semialdehyde is an important metabolite of the neurotransmitter GABA and is considered to be a physiological substrate of AKR7A2 in neuron (48). AKR7A3 has broad activity towards toxic aldehydes, but its physiological role remains to be elucidated (49). AKR1D1 is the only enzyme that catalyzes $5 \beta$-stereospecific reduction necessary for bile acid synthesis, suggesting its function in bile acid biosynthesis (46). The following section is an introduction of some important substrates of AKRs.

\section{Lipid peroxidation products}

Lipid peroxidation refers to the oxidative degradation of cellular lipids. It is a process whereby free radicals, such as reactive oxygen species (ROS), 'steal' electrons from the lipids through a chain reaction mechanism. These free radicals are generated under oxidative stress and play an important role in disease development, such as diabetic complications, Alzheimer's disease, atherosclerosis and cancer. One important feature of these radicals is that they can expand and amplify the oxidative injury through interacting with different cellular components. Membrane lipids, especially polyunsaturated fatty acids, are the main targets of these radicals and are oxidized to an array of molecules containing bioactive carbonyl groups, such as HNE and acrolein (52). $\alpha, \beta$ Unsaturated carbonyls are harmful because the aldehydic group can form a Schiff's base with the $\varepsilon$-amino group of lysines. These compounds can also form highly mutagenic etheno- and heptano-etheno-DNA adducts, inducing DNA damage and carcinogenesis (55). Interestingly, low levels of ROS and lipid peroxides, such as HNE and glutathione-conjugated HNE, act as important signals in cell growth and proliferation via a protein kinase $\mathrm{C}$ (PKC)-mediated signaling pathway (56-59). However, high HNE levels (e.g., 10$20 \mu \mathrm{M})$ trigger serious cytotoxic processes, such as genomic DNA laddering, cytochrome $c$ release from impaired mitochondrial membrane, and eventual cell death through apoptotic or necrotic pathways (60). Increased oxidative stress has been found in a variety of tumors, and HNE is the most abundant lipid peroxide (61). A rapid and effective clearance of the lipid peroxides can potentiate the tumor cell growth and proliferation.

HNE is cleared up via oxidation by aldehyde dehydrogenases to carbonic acid, reduction by reductases to alcoholic forms, or conjugation with glutathione (GSH) by glutathione $S$-transferases (GSTs). GSH-conjugates of HNE need to be further detoxified by reduction of the aldehydic group. Several enzymes are involved in the reduction of HNE and GSH-HNE, including AKR1A1, AKR1B1 and AKR1B10. Among them, AKR1B10 possesses significant physiological significance, demonstrating effective catalytic activity towards HNE at $0.10 \mu \mathrm{M}$, a concentration at or lower than physiological levels $(43,62)$. AKR1B1 also shows appreciable activity towards HNE, but is more favorable to GSH-HNE conjugates. AKR1B10 does not have enzyme activity towards GSH-HNE (43).

\section{Prostaglandins}

Prostaglandins are 20 carbon polyunsaturated fatty acid derivatives that are produced in mammalian tissues. The first step in the synthesis of prostaglandins is the conversion of arachidonic acid into prostaglandin $\mathrm{H} 2$ (PGH2) by cyclooxygenases (COX-1 and $\mathrm{COX}-2)$ and then to prostaglandin $\mathrm{D} 2$ (PGD2), prostaglandin E2 (PGE2), prostaglandin I2 (PGI2) or thromboxane A4. AKR1C3 (also named PGF synthase) catalyzes the conversion of PGH2 to PGF2 $\alpha$ and PGD2 to $9 \alpha 11 \beta$-PGF2, a biologically active stereoisomer of prostaglandin F2 $\alpha$ (PGF2 $\alpha$ ) (63). Recent studies demonstrate the 
activity of $\mathrm{AKR} 1 \mathrm{C} 1$ and $\mathrm{AKR} 1 \mathrm{C} 2$ in catalyzing the conversion of PGE2 to PGF2 $\alpha$, a physiological regulator of uterus contraction bronchoconstriction (64).

\section{Steroid aldehydes}

AKR1C proteins participate in the synthesis and metabolism of steroid hormones with differential 3-keto-, 17-keto- and 20-keto-steroid reductase activity, regulating bioactivity of sex hormones in target tissues. AKR1C1 is predominantly a HSD and reduces progesterone (a potent progestin) to $20 \alpha-$ hydroxyprogesterone (a weak progestin) (65). AKR1C2 is a $3 \alpha$-HSD and reduces $3 \alpha$-dihydrotestosterone (a potent androgen) to $3 \alpha$-androstanediol (a weak androgen), regulating ligand access to the androgen receptor in target tissues (e.g., prostate). AKR1C3 is a $17 \beta-H S D$ and converts $\Delta 4$ androstene-3,17-dione (a weak androgen) to testosterone (a potent androgen), controlling testosterone access to the androgen receptor in target tissues. AKR1C3 also reduces estrone (a weak estrogen) to $17 \beta$-estradiol (a potent estrogen) and regulates ligand access to estrogen receptor (ER) in target tissues (e.g., breast and endometrium). In addition, AKR1C3 reduces progesterone to $20 \alpha$-hydroxyprogesterone, and its dual function in the breast may produce a proestrogenic status, which could be further exacerbated by its ability to make testosterone, a substrate of aromatase $(44,66)$. Liver-specific AKR1C4 has high catalytic activity towards $3 \alpha-$ HSDs and is well suited to perform hepatic clearance of circulating steroid hormones (44).

\section{Retinaldehyde}

Retinaldehyde, i.e., retinal is the precursor of retinoic acid, a signaling molecule involved in cell growth and proliferation. Retinoic acid binds to retinoid $\mathrm{X}$ nuclear receptors and heterodimerizes with peroxisome proliferator-activated receptor $\gamma$ (PPAR $\gamma$ ) to regulate target gene transcription through the peroxisome proliferator response element, leading to an antiproliferative response and cell differentiation. In vitro, AKR1B10 displays high catalytic efficiency for the reduction of all-trans-retinal, 9-cis-retinal and 13-cis-retinal to the corresponding retinols. This conversion can deprive retinoic acid receptors of their ligand access, leading to metaplasia or dedifferentiation $(67,68)$. AKR1B1 also shows activity of reducing 9-cis-retinal with a relative low $k_{\text {cat }}$-value compared to AKR1B10 (67). This reductive activity to retinals of AKR1B1 and AKR1B10 can represent a prereceptor regulation of retinoic acid signaling.

\section{Polycyclic aromatic hydrocarbons}

PAHs are ubiquitous environmental pollutants that consist of fused aromatic rings. PAHs are present in oil, coal and tar deposits and are produced as byproducts of fuel burning, present in car exhaust, tobacco smoke and barbecued food. PAHs are considered procarcinogens and, through metabolic activation, PAHs induce carcinogenesis, such as lung cancer. AKRs (AKR1A1 and 1C1-1C4) have dihydrodiol dehydrogenase activity and convert PAHs into an extraordinarily air- sensitive chemical, catechol (69). This oxidation reaction is thermodynamically favored owing to the formation of an aromatic ring. Catechol readily yields autooxidized o-quinone through a one-electron oxidation step and producing ROS. $o$-Quinone is reactive and redox-active. In the presence of NADPH, $o$-quinone is reduced back to catechol, entering into another circle of autooxidation. This establishes a futile redox cycle, through which trace amounts of $o$-quinone can deplete cellular NADPH and amplify ROS production (70). Electrophilic and redox active $o$-quinone can also form a spectrum of DNA adducts and potentially induce p53 mutation. It has been reported that in 317 of 372 non-small cell lung carcinomas, AKR1C1/AKR1C2 was overexpressed by up to 50-fold compared to the adjacent normal tissues, indicative of poor prognosis $(71,72)$. Recently, the Penning group has revealed that AKR1B10 is also implicated in the carcinogenic activation of PAHs (73) and, more importantly, AKR$1 \mathrm{~B} 10$ expression is induced in the airway epithelium of cigarette smokers and by cigarette condensates (74). Therefore, AKR isoforms involved in the formation of PAH-o-quinones could be etiologically important in lung tumorigenesis.

\section{Anticancer drugs}

Cancer drug resistance is a sophisticated issue in cancer chemotherapy and often leads to therapeutic failure. To date, many cancer resistant mechanisms have been described, and metabolic inactivation is one of the major causes (75). Several AKR proteins such as AKR1B1 have been reported to be overexpressed in human tumors and can efficiently reduce the carbonyl group of xenobiotics to a less active alcoholic form (76). It is understood that some antitumor agents contains carbonyl groups, such as the $\mathrm{C} 13$ ketonic group in antibiotics anthracyclines; therefore, upregulated AKR proteins can induce tumor drug-resistance towards the agents bearing a carbonyl group.

Anthracyclines such as daunorubicin and doxorubicin are widely employed as chemotherapeutic agents for leukemia, lymphoma, and breast, uterine, ovarian and lung cancers. These compounds contain a C13 ketonic group and thus are potential substrates of several AKR proteins, including AKR1A1, AKR1B1, AKR1B10 and AKR1C1-4 $(75,77)$. It has been reported that the resistance of cancer cells to daunorubicin is associated with AKR1B1 and AKR1C2 expression $(76,78)$. Inhibition of AKR1B1 enhanced the cytotoxic effects of daunorubicin and doxorubicin in tumor cells (79). Similarly, mytomycin (MMC) containing active carbonyl groups is also a substrate of AKR1B1 and exposures of HepG2 cells to MMC induce AKR1B1 expression and cell resistance (80). In addition, oracin, 6-[2-(2-hydroxyethyl)aminoethyl]-5,11-dioxo-5,6-dihydro-11H-indeno [1,2-c] isoquinoline, is a new anticancer drug which is currently in Phase II clinical trials. Pharmacokinetic studies have revealed that oracin undergoes metabolic inactivation by carbonyl reduction catalyzed by the AKR1C subfamily, leading to drug resistance of cancer cells $(81,82)$.

AKRs can also play a positive role in chemotherapy. Cyclophosphamide is an active anticancer agent that is metabolically activated by cytochrome P450-mediated hydrox- 
ylation. The activated intermediate spontaneously breaks into a phosphamide mustard and acrolein. The former is the main antitumor effector by inducing the alkylating aziridinium species, whereas acrolein contributes to the dose-limiting side effect of cyclophosphamide, such as hemorrhagic cystitis. AKR1B1 and AKR1B10 efficiently catalyze the detoxification of acrolein and thus can improve the tolerance of patients to cyclophosphamide $(41,83)$.

\section{Human AKRs in carcinogenesis}

Elevated AKR expression has been found in various human tumors and could serve as a biomarker, such as AKR1B10 in smoking-related lung cancer. Although with limited understanding, it is undoubted that the study on the role of AKRs in tumor development and progression, as well as therapeutics, has become an emerging hotspot.

\section{AKR1B1}

AKR1B1 is overexpressed in liver, breast, ovarian, prostate and colorectal cancers $(58,59,84)$. Owing to its broad substrate specificity to xenobiotic carbonyls, such as lipid peroxides and antibiotic antitumor agents, AKR1B1 can promote cancer cell survival and growth by eliminating cytotoxic carbonyls and inducing drug resistance. It has been reported that AKR1B1 regulates tissue necrosis factor (TNF)$\alpha$-mediated rat vascular smooth muscle cell (VSMC) growth (85). Inhibition of AKR1B1 by small molecule inhibitors prevents high glucose- and TNF-induced activation of E2 transcription factor (E2F-1) and cyclin-dependent kinase (cdk)-2 and the expression of $\mathrm{G} 1 / \mathrm{S}$ transition regulatory proteins, such as cyclin D1, cyclin E, cdk-4, c-myc, and proliferative cell nuclear antigen. The TNF- $\alpha$-induced nuclear factor- $\kappa \mathrm{B}$ (NF-kB) activation through the PKC pathway is blocked by AKR1B1 inhibitors in these cells $(58,60)$. A similar effect was observed in Caco- 2 colon cancer cells, where AKR1B10 inhibitors attenuated fibroblast growth factor (FGF)- and platelet derived growth factor-induced cell growth through inhibition of the COX-2/PGE2 pathway (59). Therefore, AKR1B1 could be a mitogenic modulator in growth factorand inflammatory cytokine-triggered pathways.

Growth factors such as FGF, platelet-derived growth factor (PDGF), insulin-like growth factor (IGF), hepatocyte growth factor (HGF) and angiotensin, as well as cytokines such as TNF- $\alpha$ and IL-2, are inducers of oxidative stress that triggers toxic lipid peroxidation, producing unsaturated carbonyls, such as HNE. HNE can be conjugated with GSH to form GS-HNE. Both HNE and GS-HNE are efficiently converted by AKR1B1 to alcoholic forms. Exposures of VSMCs to HNE, GS-HNE or reduced GSH-1,4-dihydroxynonane, results in increase of E2F-1 expression. Inhibition of AKR1B1 prevents HNE or GS-HNE-induced but not GS-1,4-dihydroxynonane-induced upregulation of E2F-1 $(85,86)$. Conclusively, AKR1B1 activates proliferative signaling through the reduced lipid peroxides and/or their glutathione conjugates, being a mediator of ROS-initiated cytotoxic signals (Figure 3).

\section{AKR1B10}

AKR1B10, also known as ARL-1, was identified from human hepatocellular carcinoma (HCC) (39). AKR1B10 is primarily expressed in the normal colon and small intestine and thus is also named small intestine aldose reductase (68). AKR$1 \mathrm{~B} 10$ is also abundant in adrenal gland (40). Interestingly, AKR1B10 has been found to be overexpressed in multiple human cancers, such as HCC, non-small cell lung carcinomas, cervical and endometrial cancers, and is recognized as a novel tumor marker (39, 74, 87-90). In lung cancer, AKR1B10 expression is significantly associated with cigarette smoking and is involved in the activation of smoke procarcinogens, such as PAHs $(73,74)$. Our studies have shown that AKR1B10 is upregulated with tumorigenic transformation of human mammary epithelial cells and stabilizes ACCA, promoting fatty acid synthesis $(10,54)$.

AKR1B10 could contribute to cancer development and progression via several mechanisms. First, AKR1B10 is an efficient $\alpha, \beta$-unsaturated carbonyl detoxicant. These carbonyls are highly electrophilic and cytotoxic, and thus their clearance by AKR1B10 would facilitate cancer cell growth. Second, AKR1B10 efficiently converts all-trans-retinal to retinol, depleting signaling molecule retinoic acid that regulates cell proliferation and differentiation. Third, AKR1B10 is induced by cigarette smoke and activates procarcinogens PAHs in cigarette smoke, promoting malignant development of interstitial pneumonia in smokers. Fourth, many antitumor drugs contain active carbonyl groups, such as daunorubicin.

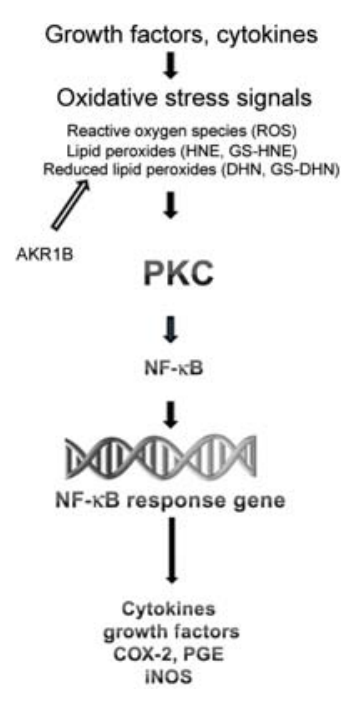

Figure 3 Involvement of AKR1B proteins in oxidative stress signaling.

Growth factors, such as FGF, PDGF, IGF, HGF, and cytokines induce intracellular oxidative stress that leads to lipid peroxidation, producing unsaturated carbonyls such as HNE and glutathione conjugates (e.g., GS-HNE). Both HNE and GS-HNE are efficiently reduced by $\mathrm{AKR} 1 \mathrm{~B} 1$ or AKR1B10 to alcoholic forms that activate protein kinase $\mathrm{C}(\mathrm{AKC}) /$ nuclear factor- $\kappa \mathrm{B}(\mathrm{NF}-\kappa \mathrm{B})$ signaling, promoting cell growth and proliferation. Therefore, AKR1B proteins could be a mediator of reactive oxygen species (ROS)-mediated signaling cascade. 
AKR1B10 could reduce the carbonyl group to less toxic alcoholic forms, inducing drug resistance to therapeutic agents $(91,92)$. Finally, data from our laboratory showed that AKR1B10, by direct association with ACCA, enhances fatty acid/lipid synthesis, which is essential for cancer cell growth and division (Figure 4) $(10,54)$. Therefore, AKR1B10 could be involved in various aspects of tumor development and progression and offer multiple advantages of tumor cell survival and proliferation, being a potential target for cancer prevention and treatment.

\section{AKR1C subfamily}

Members of the AKR1C subfamily are emerging as important mediators of cancer pathogenesis. AKR1C3 expression is increased in prostate and breast cancers (93-95). AKR$1 \mathrm{C} 3$, also known as 3-HSD or prostaglandin F synthase, is involved in steroid metabolism, including androgen, desoxycorticosterone and progesterone. It is also implicated in prostaglandin metabolism and reduction of lipid aldehydes. AKR1C3 converts 4-androstenedione to testosterone, progesterone to 20-hydroxyprogesterone and, to a lesser extent, estrone to 17-estradiol. Targeted AKR1C3 expression increased steroid conversion in MCF-7 cells and enhanced the cell growth by three times in response to estrone $(96,97)$. Therefore, a proestrogenic status induced by AKR1C 3 has been thought to be a risk factor of breast cancer.

Prostaglandins are possible promoters or growth enhancers of prostate cancer. In human prostate malignancy, elevated prostaglandin levels were detected and associated with tumor advancement. AKR1C3 has 11-ketoprostaglandin reductase activity and promotes prostate cell growth by converting PGD2 to 9,11-PGF2 $\alpha$, a proproliferative signaling molecule. Meanwhile, by producing PGF $2 \alpha$, AKR1C3 blocks the conversion of PGD2 to 15 -deoxy- $\Delta 12,14-\mathrm{PGJ} 2 \mathrm{a}$, a potential endo- genous ligand for PPAR $\gamma$. PPAR $\gamma$ activates the transcription of genes that trigger terminal differentiation and/or apoptosis $(93,98)$. Therefore, AKR1C3 acts as a switch to determine the response of prostate cells to prostaglandin signals.

Although sharing $>86 \%$ amino acid sequence similarity with AKR1C3, AKR1C2 seems to have a controversial role in prostate cancer. AKR1C2 is also upregulated in localized and advanced prostate cancer, and treatment of PC3-AKR1C2 cells with PGD2 increased cell proliferation by activating the PI3K/Akt pathway (99). On the contrary, another laboratory found that both AKR1C2 and AKR1C1 expression in prostate cancer was decreased compared to paired benign tissues, leading to androgen-dependent cellular proliferation by increased dihydrotestosterone that is eliminated by AKR1C2 (100).

In addition, AKR1C isoforms were also found to be upregulated dramatically in lung cancer and could be implicated in lung carcinogenesis by activating environmental pollutant PAHs to catechol, a carcinogen (71).

\section{Conclusion}

AKR proteins are conserved in evolutionary hierarchy of organisms and are featured with a wide range of substrate specificity. In the nomenclature system, AKR proteins are grouped into different family and subfamily groups according to the sequence similarity. AKR members that are expressed in human fall into AKR1, AKR6 and AKR7 families; and these human isoforms play wide pathophysiological roles, including carbonyl detoxification, hormone metabolism, fatty acid/lipid synthesis, osmolytic regulation, diabetic complications, procarcinogen activation and anticancer drug resistance. Growing evidence also indicates that AKRs extensively participate in oxidative stress regulation and nuclear

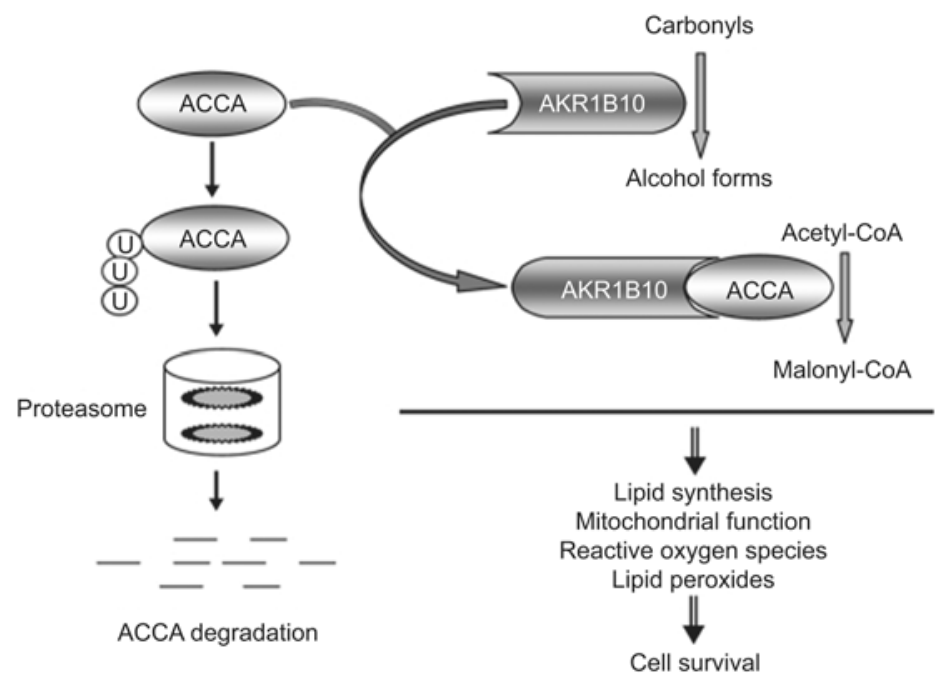

Figure 4 Hypothetical model of AKR1B10 in regulating cell growth and survival.

Acetyl-CoA carboxylase- $\alpha$ (ACCA) is degraded through the ubiquitination-proteasome pathway. AKR1B10 blocks ubiquitin-dependent degradation of the ACCA and thus enhances fatty acid/lipid synthesis, which affects mitochondrial function, oxidative status and lipid peroxidation of cells. AKR1B10 also reduces electrophilic carbonyls to less toxic alcohol forms, blocking their cytotoxicity. 
receptor signaling (such as prostaglandins, retinoic acid and steroid hormones). In view of their overexpression in human tumors, therefore, AKRs could be profoundly implicated in cancer development and progression, and thus are potential tumor markers and novel targets for cancer intervention.

\section{References}

1. Hyndman D, Bauman DR, Heredia VV, Penning TM. The aldo-keto reductase superfamily homepage. Chem Biol Interact 2003; 143-144: 621-31.

2. Seery LT, Nestor PV, FitzGerald GA. Molecular evolution of the aldo-keto reductase gene superfamily. J Mol Evol 1998; 46: 139-46.

3. Bohren KM, Bullock B, Wermuth B, Gabbay KH. The aldoketo reductase superfamily. cDNAs and deduced amino acid sequences of human aldehyde and aldose reductases. J Biol Chem 1989; 264: 9547-51.

4. Jez JM, Flynn TG, Penning TM. A nomenclature system for the aldo-keto reductase superfamily. Adv Exp Med Biol 1997; 414: 579-600.

5. Fujii Y, Watanabe K, Hayashi H, Urade Y, Kuramitsu S, Kagamiyama $\mathrm{H}$, Hayaishi O. Purification and characterization of rho-crystallin from Japanese common bullfrog lens. J Biol Chem 1990; 265: 9914-23.

6. van Boekel MA, van Aalten DM, Caspers GJ, Roll B, de Jong WW. Evolution of the aldose reductase-related gecko eye lens protein rhoB-crystallin: a sheep in wolf's clothing. J Mol Evol 2001; 52: 239-48.

7. Weng J, Cao Y, Moss N, Zhou M. Modulation of voltagedependent Shaker family potassium channels by an aldo-keto reductase. J Biol Chem 2006; 281: 15194-200.

8. Jez JM, Bennett MJ, Schlegel BP, Lewis M, Penning TM. Comparative anatomy of the aldo-keto reductase superfamily. Biochem J 1997; 326: 625-36.

9. Rondeau JM, Tete-Favier F, Podjarny A, Reymann JM, Barth $\mathrm{P}$, Biellmann JF, Moras D. Novel NADPH-binding domain revealed by the crystal structure of aldose reductase. Nature 1992; 355: 469-72.

10. Ma J, Yan R, Zu X, Cheng JM, Rao K, Liao DF, Cao D. Aldoketo reductase family $1 \mathrm{~B} 10$ affects fatty acid synthesis by regulating the stability of acetyl-CoA carboxylase- $\alpha$ in breast cancer cells. J Biol Chem 2008; 283: 3418-23.

11. Vega MC, Lorentzen E, Linden A, Wilmanns M. Evolutionary markers in the $(\beta / \alpha) 8$-barrel fold. Curr Opin Chem Biol 2003; 7: 694-701.

12. Jurgens C, Strom A, Wegener D, Hettwer S, Wilmanns M, Sterner R. Directed evolution of a $(\beta \alpha)$ 8-barrel enzyme to catalyze related reactions in two different metabolic pathways. Proc Natl Acad Sci USA 2000; 97: 9925-30.

13. Wilson DK, Bohren KM, Gabbay KH, Quiocho FA. An unlikely sugar substrate site in the $1.65 \AA$ structure of the human aldose reductase holoenzyme implicated in diabetic complications. Science 1992; 257: 81-4.

14. Bennett MJ, Schlegel BP, Jez JM, Penning TM, Lewis M. Structure of $3 \alpha$-hydroxysteroid/dihydrodiol dehydrogenase complexed with NADP ${ }^{+}$. Biochemistry 1996; 35: 10702-11.

15. Ma H, Ratnam K, Penning TM. Mutation of nicotinamide pocket residues in rat liver $3 \alpha$-hydroxysteroid dehydrogenase reveals different modes of cofactor binding. Biochemistry 2000; 39: 102-9.
16. Lefrancois-Martinez AM, Tournaire C, Martinez A, Berger M, Daoudal S, Tritsch D, Veyssiere G, Jean C. Product of sidechain cleavage of cholesterol, isocaproaldehyde, is an endogenous specific substrate of mouse vas deferens protein, an aldose reductase-like protein in adrenocortical cells. J Biol Chem 1999; 274: 32875-80.

17. Liu SQ, Bhatnagar A, Srivastava SK. Bovine lens aldose reductase. $\mathrm{pH}$-dependence of steady-state kinetic parameters and nucleotide binding. J Biol Chem 1993; 268: 25494-9.

18. Bohren KM, Page JL, Shankar R, Henry SP, Gabbay KH. Expression of human aldose and aldehyde reductases. Sitedirected mutagenesis of a critical lysine 262. J Biol Chem 1991; 266: 24031-7.

19. Schlegel BP, Ratnam K, Penning TM. Retention of NADPHlinked quinone reductase activity in an aldo-keto reductase following mutation of the catalytic tyrosine. Biochemistry 1998; 37: 11003-11.

20. Schlegel BP, Jez JM, Penning TM. Mutagenesis of $3 \alpha-$ hydroxysteroid dehydrogenase reveals a "push-pull" mechanism for proton transfer in aldo-keto reductases. Biochemistry 1998; 37: 3538-48.

21. Lee YS, Hodoscek M, Brooks BR, Kador PF. Catalytic mechanism of aldose reductase studied by the combined potentials of quantum mechanics and molecular mechanics. Biophys Chem 1998; 70: 203-16.

22. Ye Q, Hyndman D, Green N, Li X, Korithoski B, Jia Z, Flynn TG. Crystal structure of an aldehyde reductase Y50F mutantNADP complex and its implications for substrate binding. Proteins 2001 ; 44: 12-9.

23. Grimshaw CE, Shahbaz M, Putney CG. Mechanistic basis for nonlinear kinetics of aldehyde reduction catalyzed by aldose reductase. Biochemistry 1990; 29: 9947-55.

24. Grimshaw CE, Bohren KM, Lai CJ, Gabbay KH. Human aldose reductase: rate constants for a mechanism including interconversion of ternary complexes by recombinant wildtype enzyme. Biochemistry 1995; 34: 14356-65.

25. Bohren KM, Grimshaw CE, Lai CJ, Harrison DH, Ringe D, Petsko GA, Gabbay KH. Tyrosine-48 is the proton donor and histidine-110 directs substrate stereochemical selectivity in the reduction reaction of human aldose reductase: enzyme kinetics and crystal structure of the $\mathrm{Y} 48 \mathrm{H}$ mutant enzyme. Biochemistry 1994; 33: 2021-32.

26. Grimshaw CE, Bohren KM, Lai CJ, Gabbay KH. Human aldose reductase: $\mathrm{pK}$ of tyrosine 48 reveals the preferred ionization state for catalysis and inhibition. Biochemistry 1995; 34: 14374-84.

27. Jin Y, Penning TM. Multiple steps determine the overall rate of the reduction of $5 \alpha$-dihydrotestosterone catalyzed by human type $33 \alpha$-hydroxysteroid dehydrogenase: implications for the elimination of androgens. Biochemistry 2006; 45: 13054-63.

28. Heredia VV, Penning TM. Dissection of the physiological interconversion of $5 \alpha$-DHT and $3 \alpha$-diol by rat $3 \alpha-$ HSD via transient kinetics shows that the chemical step is rate-determining: effect of mutating cofactor and substrate-binding pocket residues on catalysis. Biochemistry 2004; 43: 12028-37.

29. Urzhumtsev A, Tete-Favier F, Mitschler A, Barbanton J, Barth P, Urzhumtseva L, Biellmann JF, Podjarny A, Moras DA. 'Specificity' pocket inferred from the crystal structures of the complexes of aldose reductase with the pharmaceutically important inhibitors tolrestat and sorbinil. Structure 1997; 5: 601-12.

30. Harrison DH, Bohren KM, Ringe D, Petsko GA, Gabbay KH. An anion binding site in human aldose reductase: mechanistic 
implications for the binding of citrate, cacodylate, glucose 6phosphate. Biochemistry 1994; 33: 2011-20.

31. Pfeifer MA, Schumer MP, Gelber DA. Aldose reductase inhibitors: the end of an era or the need for different trial designs? Diabetes 1997; 46 (Suppl 2): S82-9.

32. Ramirez MA, Borja NL. Epalrestat: an aldose reductase inhibitor for the treatment of diabetic neuropathy. Pharmacotherapy 2008; 28: 646-55.

33. Wermuth B, Munch JD, von Wartburg JP. Purification and properties of NADPH-dependent aldehyde reductase from human liver. J Biol Chem 1977; 252: 3821-8.

34. Petrash JM, Srivastava SK. Purification and properties of human liver aldehyde reductases. Biochim Biophys Acta 1982; 707: 105-14.

35. Barski OA, Papusha VZ, Ivanova MM, Rudman DM, Finegold MJ. Developmental expression and function of aldehyde reductase in proximal tubules of the kidney. Am J Physiol Renal Physiol 2005; 289: F200-7.

36. O'Connor T, Ireland LS, Harrison DJ, Hayes JD. Major differences exist in the function and tissue-specific expression of human aflatoxin B1 aldehyde reductase and the principal human aldo-keto reductase AKR1 family members. Biochem J 1999; 343: 487-504.

37. Dvornik E, Simard-Duquesne N, Krami M, Sestanj K, Gabbay KH, Kinoshita JH, Varma SD, Merola LO. Polyol accumulation in galactosemic and diabetic rats: control by an aldose reductase inhibitor. Science 1973; 182: 1146-8.

38. Gabbay KH, Merola LO, Field RA. Sorbitol pathway: presence in nerve and cord with substrate accumulation in diabetes. Science 1966; 151: 209-10.

39. Cao D, Fan ST, Chung SS. Identification and characterization of a novel human aldose reductase-like gene. J Biol Chem 1998; 273: 11429-35.

40. Hyndman DJ, Flynn TG. Sequence and expression levels in human tissues of a new member of the aldo-keto reductase family. Biochim Biophys Acta 1998; 1399: 198-202.

41. Zu X, Yan R, Robbins S, Krishack PA, Liao DF, Cao D. Reduced 293T cell susceptibility to acrolein due to aldose reductase-like-1 protein expression. Toxicol Sci 2007; 97: 562-8.

42. Yan R, Zu X, Ma J, Liu Z, Adeyanju M, Cao D. Aldo-keto reductase family $1 \mathrm{~B} 10$ gene silencing results in growth inhibition of colorectal cancer cells: implication for cancer intervention. Int J Cancer 2007; 121: 2301-6.

43. Zhong L, Liu Z, Yan R, Johnson, S, Zhao Y, Fang X, Cao D. Aldo-keto reductase family $1 \mathrm{~B} 10$ protein detoxifies dietary and lipid-derived $\alpha, \beta$-unsaturated carbonyls at physiological levels. Biochem Biophys Res Commun 2009; 387: 245-50.

44. Penning TM, Jin Y, Steckelbroeck S, Lanisnik Rizner T, Lewis M. Structure-function of human $3 \alpha$-hydroxysteroid dehydrogenases: genes and proteins. Mol Cell Endocrinol 2004; 215: 63-72.

45. Penning TM, Jin Y, Heredia VV, Lewis M. Structure-function relationships in $3 \alpha$-hydroxysteroid dehydrogenases: a comparison of the rat and human isoforms. J Steroid Biochem Mol Biol 2003; 85: 247-55.

46. Okuda A, Okuda K. Purification and characterization of $84-3-$ ketosteroid 5 $\beta$-reductase. J Biol Chem 1984; 259: 7519-24.

47. Mode A, Rafter I. The sexually differentiated $\delta 4-3$-ketosteroid $5 \beta$-reductase of rat liver. Purification, characterization, quantitation. J Biol Chem 1985; 260: 7137-41.

48. Lyon RC, Johnston SM, Watson DG, McGarvie G, Ellis EM. Synthesis and catabolism of $\gamma$-hydroxybutyrate in SH-SY5Y human neuroblastoma cells: role of the aldo-keto reductase AKR7A2. J Biol Chem 2007; 282: 25986-92.
49. Knight LP, Primiano T, Groopman JD, Kensler TW, Sutter TR. cDNA cloning, expression and activity of a second human aflatoxin B1-metabolizing member of the aldo-keto reductase superfamily, AKR7A3. Carcinogenesis 1999; 20: 1215-23.

50. Picklo MJ, Montine TJ, Amarnath V, Neely MD. Carbonyl toxicology and Alzheimer's disease. Toxicol Appl Pharmacol 2002; 184: 187-97.

51. Brownlee M. Biochemistry and molecular cell biology of diabetic complications. Nature 2001; 414: 813-20.

52. Negre-Salvayre A, Coatrieux C, Ingueneau C, Salvayre R. Advanced lipid peroxidation end products in oxidative damage to proteins. Potential role in diseases and therapeutic prospects for the inhibitors. Br J Pharmacol 2008; 153: 6-20.

53. Petrash JM. All in the family: aldose reductase and closely related aldo-keto reductases. Cell Mol Life Sci 2004; 61: 73749.

54. Wang C, Yan R, Luo D, Watabe K, Liao DF, Cao D. Aldoketo reductase family 1 member B10 promotes cell survival by regulating lipid synthesis and eliminating carbonyls. J Biol Chem 2009; 284: 26742-8.

55. Ichihashi K, Osawa T, Toyokuni S, Uchida K. Endogenous formation of protein adducts with carcinogenic aldehydes: implications for oxidative stress. J Biol Chem 2001; 276: 23903-13.

56. Srivastava SK, Ramana KV, Bhatnagar A. Role of aldose reductase and oxidative damage in diabetes and the consequent potential for therapeutic options. Endocr Rev 2005; 26: 38092.

57. Temma K, Shimoya K, Zhang Q, Kimura T, Wasada K, Kanzaki T, Azuma C, Koyama M, Murata Y. Effects of 4-hydroxy2-nonenal, a marker of oxidative stress, on the cyclooxygenase-2 of human placenta in chorioamnionitis. Mol Hum Reprod 2004; 10: 167-71.

58. Tammali R, Ramana KV, Singhal SS, Awasthi S, Srivastava SK. Aldose reductase regulates growth factor-induced cyclooxygenase-2 expression and prostaglandin E2 production in human colon cancer cells. Cancer Res 2006; 66: 9705-13.

59. Tammali R, Ramana KV, Srivastava SK. Aldose reductase regulates TNF- $\alpha$-induced PGE2 production in human colon cancer cells. Cancer Lett 2007; 252: 299-306.

60. Ramana KV, Bhatnagar A, Srivastava S, Yadav UC, Awasthi S, Awasthi YC, Srivastava SK. Mitogenic responses of vascular smooth muscle cells to lipid peroxidation-derived aldehyde 4-hydroxy-trans-2-nonenal (HNE): role of aldose reductase-catalyzed reduction of the HNE-glutathione conjugates in regulating cell growth. J Biol Chem 2006; 281: 17652-60.

61. Behrend L, Henderson G, Zwacka RM. Reactive oxygen species in oncogenic transformation. Biochem Soc Trans 2003; 31: 1441-4.

62. Srivastava S, Chandra A, Bhatnagar A, Srivastava SK, Ansari NH. Lipid peroxidation product, 4-hydroxynonenal and its conjugate with GSH are excellent substrates of bovine lens aldose reductase. Biochem Biophys Res Commun 1995; 217: 741-6.

63. Suzuki-Yamamoto T, Nishizawa M, Fukui M, Okuda-Ashitaka E, Nakajima T, Ito S, Watanabe K. cDNA cloning, expression and characterization of human prostaglandin F synthase. FEBS Lett 1999; 462: 335-40.

64. Dozier BL, Watanabe K, Duffy DM. Two pathways for prostaglandin F2 $\alpha$ synthesis by the primate periovulatory follicle. Reproduction 2008; 136: 53-63.

65. Vergnes L, Phan J, Stolz A, Reue K. A cluster of eight hydroxysteroid dehydrogenase genes belonging to the aldoketo reductase supergene family on mouse chromosome 13 . J Lipid Res 2003; 44: 503-11. 
66. Penning TM, Burczynski ME, Jez JM, Hung CF, Lin HK, Ma H, Moore M, Palackal N, Ratnam K. Human 3alpha-hydroxysteroid dehydrogenase isoforms (AKR1C1-AKR1C4) of the aldo-keto reductase superfamily: functional plasticity and tissue distribution reveals roles in the inactivation and formation of male and female sex hormones. Biochem J 2000; 351: $67-77$.

67. Ruiz FX, Gallego O, Ardevol A, Moro A, Dominguez M, Alvarez S, Alvarez R, de Lera AR, Rovira C, Fita I, Pares X, Farres J. Aldo-keto reductases from the AKR1B subfamily: retinoid specificity and control of cellular retinoic acid levels. Chem Biol Interact 2009; 178: 171-7.

68. Crosas B, Hyndman DJ, Gallego O, Martras S, Pares X, Flynn TG, Farres J. Human aldose reductase and human small intestine aldose reductase are efficient retinal reductases: consequences for retinoid metabolism. Biochem J 2003; 373: 973-9.

69. Penning TM, Burczynski ME, Hung CF, McCoull KD, Palackal NT, Tsuruda LS. Dihydrodiol dehydrogenases and polycyclic aromatic hydrocarbon activation: generation of reactive and redox active o-quinones. Chem Res Toxicol 1999; 12: 1-18.

70. Penning TM, Ohnishi ST, Ohnishi T, Harvey RG. Generation of reactive oxygen species during the enzymatic oxidation of polycyclic aromatic hydrocarbon trans-dihydrodiols catalyzed by dihydrodiol dehydrogenase. Chem Res Toxicol 1996; 9: 84-92.

71. Palackal NT, Lee SH, Harvey RG, Blair IA, Penning TM. Activation of polycyclic aromatic hydrocarbon trans-dihydrodiol proximate carcinogens by human aldo-keto reductase (AKR1C) enzymes and their functional overexpression in human lung carcinoma (A549) cells. J Biol Chem 2002; 277: 24799-808.

72. Hsu NY, Ho HC, Chow KC, Lin TY, Shih CS, Wang LS, Tsai $\mathrm{CM}$. Overexpression of dihydrodiol dehydrogenase as a prognostic marker of non-small cell lung cancer. Cancer Res 2001; 61: 2727-31.

73. Quinn AM, Harvey RG, Penning TM. Oxidation of PAH transdihydrodiols by human aldo-keto reductase AKR1B10. Chem Res Toxicol 2008; 21: 2207-15.

74. Fukumoto S, Yamauchi N, Moriguchi H, Hippo Y, Watanabe A, Shibahara J, Taniguchi H, Ishikawa S, Ito H, Yamamoto S, Iwanari H, Hironaka M, Ishikawa Y, Niki T, Sohara Y, Kodama T, Nishimura M, Fukayama M, Dosaka-Akita H, Aburatani $\mathrm{H}$. Overexpression of the aldo-keto reductase family protein AKR1B10 is highly correlated with smokers' non-small cell lung carcinomas. Clin Cancer Res 2005; 11: 1776-85.

75. Barski OA, Tipparaju SM, Bhatnagar A. The aldo-keto reductase superfamily and its role in drug metabolism and detoxification. Drug Metab Rev 2008; 40: 553-624.

76. Lee KW, Ko BC, Jiang Z, Cao D, Chung SS. Overexpression of aldose reductase in liver cancers may contribute to drug resistance. Anticancer Drugs 2001; 12: 129-32.

77. Jin Y, Penning TM. Aldo-keto reductases and bioactivation/ detoxication. Annu Rev Pharmacol Toxicol 2007; 47: 263-92.

78. Ax W, Soldan M, Koch L, Maser E. Development of daunorubicin resistance in tumour cells by induction of carbonyl reduction. Biochem Pharmacol 2000; 59: 293-300.

79. Lee EK, Regenold WT, Shapiro P. Inhibition of aldose reductase enhances HeLa cell sensitivity to chemotherapeutic drugs and involves activation of extracellular signal-regulated kinases. Anticancer Drugs 2002; 13: 859-68.

80. Yang XD, Tang DN, Wang J, Cao DL. [Screening of the drug resistance-associated gene in HepG2 cell line transfected with aldose reductase like gene-1 (ARL-1)]. Ai Zheng 2003; 22: 1289-95 (in Chinese).
81. Wsol V, Szotakova B, Martin HJ, Maser E. Aldo-keto reductases (AKR) from the AKR1C subfamily catalyze the carbonyl reduction of the novel anticancer drug oracin in man. Toxicology 2007; 238: 111-8.

82. Novotna R, Wsol V, Xiong G, Maser E. Inactivation of the anticancer drugs doxorubicin and oracin by aldo-keto reductase (AKR) 1C3. Toxicol Lett 2008; 181: 1-6.

83. Kolb NS, Hunsaker LA, Vander Jagt DL. Aldose reductasecatalyzed reduction of acrolein: implications in cyclophosphamide toxicity. Mol Pharmacol 1994; 45: 797-801.

84. Saraswat M, Mrudula T, Kumar PU, Suneetha A, Rao Rao TS, Srinivasulu M, Reddy B. Overexpression of aldose reductase in human cancer tissues. Med Sci Monit 2006; 12: CR525-9.

85. Tammali R, Saxena A, Srivastava SK, Ramana KV. Aldose reductase regulates vascular smooth muscle cell proliferation by modulating G1/S phase transition of cell cycle. Endocrinology 2010; 151: 2140-50.

86. Ruef J, Liu SQ, Bode C, Tocchi M, Srivastava S, Runge MS, Bhatnagar A. Involvement of aldose reductase in vascular smooth muscle cell growth and lesion formation after arterial injury. Arterioscler Thromb Vasc Biol 2000; 20: 1745-52.

87. Balendiran GK, Martin HJ, El-Hawari Y, Maser E. Cancer biomarker AKR1B10 and carbonyl metabolism. Chem Biol Interact 2009; 178: 134-7.

88. Li CP, Goto A, Watanabe A, Murata K, Ota S, Niki T, Aburatani $\mathrm{H}$, Fukayama M. AKR1B10 in usual interstitial pneumonia: expression in squamous metaplasia in association with smoking and lung cancer. Pathol Res Pract 2008; 204: 295304.

89. Penning TM. AKR1B10: a new diagnostic marker of nonsmall cell lung carcinoma in smokers. Clin Cancer Res 2005; 11: $1687-90$.

90. Yoshitake H, Takahashi M, Ishikawa H, Nojima M, Iwanari H, Watanabe A, Aburatani H, Yoshida K, Ishi K, Takamori K, Ogawa H, Hamakubo T, Kodama T, Araki Y. Aldo-keto reductase family 1, member B10 in uterine carcinomas: a potential risk factor of recurrence after surgical therapy in cervical cancer. Int J Gynecol Cancer 2007; 17: 1300-6.

91. Martin HJ, Breyer-Pfaff U, Wsol V, Venz S, Block S, Maser E. Purification and characterization of akr1b10 from human liver: role in carbonyl reduction of xenobiotics. Drug Metab Dispos 2006; 34: 464-70.

92. Balendiran GK. Fibrates in the chemical action of daunorubicin. Curr Cancer Drug Targets 2009; 9: 366-9.

93. Huang KH, Chiou SH, Chow KC, Lin TY, Chang HW, Chiang IP, Lee MC. Overexpression of aldo-keto reductase $1 \mathrm{C} 2$ is associated with disease progression in patients with prostatic cancer. Histopathology 2010; 57: 384-94.

94. Tai HL, Lin TS, Huang HH, Lin TY, Chou MC, Chiou SH, Chow KC. Overexpression of aldo-keto reductase $1 \mathrm{C} 2$ as a high-risk factor in bladder cancer. Oncol Rep 2007; 17: 305-11.

95. Dozmorov MG, Azzarello JT, Wren JD, Fung KM, Yang Q, Davis JS, Hurst RE, Culkin DJ, Penning TM, Lin HK. Elevated AKR1C3 expression promotes prostate cancer cell survival and prostate cell-mediated endothelial cell tube formation: implications for prostate cancer progression. BMC Cancer 2010; 10: 672.

96. Byrns MC, Penning TM. Type 5 17beta-hydroxysteroid dehydrogenase/prostaglandin F synthase (AKR1C3): role in breast cancer and inhibition by non-steroidal anti-inflammatory drug analogs. Chem Biol Interact 2009; 178: 221-7.

97. Kang KW, Kim YG. Bioequivalence studies of tibolone in premenopausal women and effects on expression of the tibolone- 
metabolizing enzyme AKR1C (aldo-keto reductase) family caused by estradiol. J Clin Pharmacol 2008; 48: 1430-7.

98. Fung KM, Samara EN, Wong C, Metwalli A, Krlin R, Bane B, Liu CZ, Yang JT, Pitha JV, Culkin DJ, Kropp BP, Penning TM, Lin HK. Increased expression of type $23 \alpha$-hydroxysteroid dehydrogenase/type 5 17ß-hydroxysteroid dehydrogenase (AKR1C3) and its relationship with androgen receptor in prostate carcinoma. Endocr Relat Cancer 2006; 13: 169-80.
99. Rizner TL, Lin HK, Penning TM. Role of human type $33 \alpha$ hydroxysteroid dehydrogenase (AKR1C2) in androgen metabolism of prostate cancer cells. Chem Biol Interact 2003; 143144: 401-9.

100. Wang S, Yang Q, Fung KM, Lin HK. AKR1C2 and AKR1C3 mediated prostaglandin D2 metabolism augments the PI3K/ Akt proliferative signaling pathway in human prostate cancer cells. Mol Cell Endocrinol 2008; 289: 60-6. 